\title{
A seven-year surveillance study of the epidemiology, antifungal susceptibility, risk factors and mortality of candidaemia among paediatric and adult inpatients in a tertiary teaching hospital in China
}

\author{
Zhangrui Zeng, Yinhuan Ding, Gang Tian, Kui Yang, Jian Deng, Guangrong Li and Jinbo Liu* (D)
}

\begin{abstract}
Background: There are no current national estimates of the candidaemia burden in China, and epidemiological candidaemia data from the underdeveloped region of China are lacking.

Methods: A 7-year retrospective study was carried out to analyse the prevalence, species distribution, antifungal susceptibility, risk factors and inpatient mortality of candidaemia among paediatric and adult patients in a regional tertiary teaching hospital in China.

Results: During the seven-year study period, a total of 201 inpatients with candidaemia were identified. The median age of the patients was 65 years (range, 1 day to 92 years), and 114 of the patients (56.7\%) were male. The mean annual incidence of candidaemia was 0.26 cases per 1000 admissions ( 0.42 cases per 1000 paediatric admissions vs 0.24 cases per 1000 adult admissions, $P<0.05)$. Candida albicans was the most common fungal species $(81 / 201,40.3 \%)$ in all patients, Candida glabrata was the most common fungal species $(18 / 35,51.4 \%)$ in paediatric patients. Most isolates were susceptible to flucytosine (99.0\%) and amphotericin B (99.0\%), and the activity of antifungal agents against Candida species was no significant difference in satisfaction between paediatric and adult patients $(P>0.05)$. The all-cause mortality rate was $20.4 \%$ (paediatric patients: $11.4 \%$ vs adult patients: $22.3 \%, P>0.05)$. Fewer univariate predictors of poor outcomes were identified for paediatric patients than for adult patients (4 vs 11 predictors). Respiratory dysfunction and septic shock were independent predictors of 30-day mortality for all patients.

Conclusions: The epidemiological data of candidaemia in paediatric and adult patients are only different in the distributions of Candida species and the mean annual incidence of candidaemia. Flucytosine and amphotericin B can be used as first-choice agents when no antifungal susceptibility test results are available.
\end{abstract}

Keywords: Candidaemia, Epidemiology, Paediatric patients, Adult patients, Risk factors

*Correspondence: liulab2019@163.com

Department of Laboratory Medicine, the Affiliated Hospital of Southwest

Medical University, 25 Taiping street, Luzhou 646000, P.R. China

C C The Author(s). 2020 Open Access This article is licensed under a Creative Commons Attribution 4.0 International License, which permits use, sharing, adaptation, distribution and reproduction in any medium or format, as long as you give appropriate credit to the original author(s) and the source, provide a link to the Creative Commons licence, and indicate if changes were made. The images or other third party material in this article are included in the article's Creative Commons licence, unless indicated otherwise in a credit line to the material. If material is not included in the article's Creative Commons licence and your intended use is not permitted by statutory regulation or exceeds the permitted use, you will need to obtain permission directly from the copyright holder. To view a copy of this licence, visit http://creativecommons.org/licenses/by/4.0/ The Creative Commons Public Domain Dedication waiver (http://creativecommons.org/publicdomain/zero/1.0/) applies to the data made available in this article, unless otherwise stated in a credit line to the data. 


\section{Background}

Candidaemia is the most common fungal disease among hospitalised patients worldwide and is the fourth to tenth most common bloodstream infection (BSI) in most population-based studies [1, 2]. Candidaemia is associated with significant morbidity and mortality [3]. The main risk factors for candidaemia include critical illness, a long intensive care unit (ICU) length of stay, haematologic malignant disease, solid-organ transplantation, solid-organ tumours, low birth weight in neonates and preterm infants, broad-spectrum antimicrobial agent use, central venous catheterization (CVC), total parenteral nutrition, haemodialysis, abdominal surgery, and aggressive chemotherapy [1]. With the increase in related research, reports have shown that the incidence of candidaemia is age-specific, with maximum rates observed in those with older age (over 65 years) [1, 4, 5].

More than 40 Candida species can cause candidaemia in humans [6]. Five species of Candida (Candida albicans, Candida glabrata, Candida parapsilosis, Candida tropicalis and Candida krusei) are the most common species and account for more than $90 \%$ of all the isolates [2]. The variability in the relative proportions of Candida isolates has been associated with clinical condition or risk factors such as age, underlying comorbidities, the extensive use of antifungal agents and geography. Candida albicans is the primary cause of candidaemia and one of the most common species in many countries, Candida glabrata is the second or third most common species in the USA and Europe, and Candida parapsilosis is predominant in neonates in South America, southern Europe and Asia [2]. The global incidence of candidaemia varies from 0.3 to 5 per 1000 admissions according to geographical region, local epidemiology, age and other factors [7]; the 30-day mortality among all patients with candidaemia has been reported to be between 22 and $70 \%$ [8], and the cost of candidaemia treatment has been reported to be US $\$ 40,000$ per patient $[1,9,10]$.

In China, the epidemiology of candidaemia varies widely among different areas [11]. Epidemiological surveillance of candidaemia has focused on ICUs and single centres in China, and national surveillance systems are usually absent. Most of the existing epidemiological surveillance of candidaemia has focused on adults or children, and little information about general populations (including neonates, children and adults) is known. Therefore, in the present study, we performed a seven-year retrospective study to evaluate the epidemiology, antifungal susceptibility, risk factors and mortality of candidaemia among all inpatients in a tertiary teaching hospital in China.

\section{Methods}

\section{Patient data collection}

We conducted a retrospective observational study of electronic laboratory records. The fungal specimen data were collected from inpatients with candidaemia in the Affiliated Hospital of Southwest Medical University (Luzhou, China), which is a 3200-bed tertiary care teaching hospital with 43 wards and approximately 120,000 annual admissions, from January 2013 to December 2019. The diagnostic criteria of candidaemia were based on the guidelines for the diagnosis and treatment of Candidiasis: the expert consensus issued by the Chinese Medical Association [12]; these criteria were also in accordance with the European Society of Clinical Microbiology and Infectious Diseases (ESCMID)* guidelines for the diagnosis and management of Candida diseases 2012 [13, 14] and the Infectious Diseases Society of America (IDSA) Guidelines for the Management of Candidiasis: 2016 Update [15]. For each patient, only the first episode was included in our analysis. Patient cultures with two or more Candida species were excluded from the analysis, and all data were collected from electronic medical records. The following data were retrospectively collected from all patients: demographic characteristics, underlying comorbidities, Candida species, susceptibility to antifungal agents and mortality. Data on the following risk factors associated with candidaemia were also collected: gestational age and weight of neonates, indwelling central vascular catheter, mechanical ventilation, systemic corticosteroid treatment (a dose equivalent to prednisone $10 \mathrm{mg} / \mathrm{d}$ for at least 14 days), total parenteral nutrition, chemotherapy, abdominal surgery, ICU admission, neutropenia (absolute neutrophil count $<500$ cells $/ \mu \mathrm{l})$, concomitant bacterial infections, septic shock, haemodialysis, broad-spectrum antibiotic use and treatment with antifungal agents. The study protocol was approved by the ethics committee of the hospital (Project No. KY2020043). The need for informed consent was waived by the Clinical Research Ethics Committee.

\section{Microorganism identification and antifungal susceptibility} According to the manufacturer's instructions, blood was inoculated into both aerobic and anaerobic BacT/AlerT 3D vials (Bruker Diagnostics Inc., USA). All positive cultures were manually sampled and inoculated onto CHROMagar Candida medium (CHROMagar Company, France) to ensure viability and purity. The identification of all species was confirmed by a MicroScan WalkAway 96 Plus System (Siemens, Germany) and Microflex LT (Bruker Diagnostics Inc., USA) matrix-assisted laser-desorption/ionization timeof-flight (MALDI-TOF) mass spectrometry (MS) system.

Antifungal susceptibility tests for fluconazole (FCA), itraconazole (ITR), voriconazole (VRC), flucytosine (5FC) and amphotericin B (AMB) were performed for all Candida strain isolates by using an ATB FUNGUS 3 kit (bioMérieux, France). The minimal inhibitory concentrations (MICs) of the antifungal agents were judged by 
visualization in our laboratory according to the manufacturer's instructions. The quality control strains were $C$. parapsilosis ATCC 22019 and C. krusei ATCC 6258. The results were interpreted using the Clinical and Laboratory Standards Institute M27-A3 microbroth dilution method.

\section{Statistical analyses}

The data were analysed using Microsoft Excel (version 2016, Redmond, USA) and IBM SPSS software version 24 for Windows (IBM, Armonk, USA). Categorical data were compared using chi-square or Fisher's exact tests. Continuous data were analysed using Student's t-test or the Mann-Whitney U test. Multivariable logistic regression analysis was performed to identify independent predictors of candidemia and 30-day hospital mortality. Biologically plausible variables with a value of $P<0.1$ according to the univariate analyses were included in the multiple logistic regression model. Statistical significance was determined using two-tailed tests, and $P<0.05$ was considered statistically significant.

\section{Results}

A total of 201 distinct candidaemia episodes were identified during our study period. The median age was 65 years (range 1 day - 92 years), and 114 patients (56.7\%) were male. Most candidaemia episodes were diagnosed in medical wards $(89,44.3 \%)$, followed by ICUs (46, $22.9 \%)$, paediatric wards $(35,17.4 \%)$ and surgical wards (31, 15.4\%). Most of the patients had one or more comorbidities. Pulmonary infection (49.8\%), chronic/acute renal failure (45.3\%) and cardiovascular disease (42.8\%) were the most common underlying comorbidities, followed by neurological diseases $(38.8 \%)$, diabetes mellitus (29.9\%), respiratory dysfunction (28.9\%), gastrointestinal pathologies $(28.9 \%)$ and chronic/acute liver disease (24.4\%). Moreover, the most common underlying conditions documented prior to candidaemia were prior exposure to broad-spectrum antibiotics $(89.1 \%)$, treatment with antifungal agents (56.7\%), concomitant bacterial infections (54.7), total parenteral nutrition (47.3\%), mechanical ventilation (43.3\%), ICU/paediatric ICU (PICU)/ neonatal ICU (NICU) admission (40.3\%) and CVC (38.3\%). In total, $53(26.4 \%, 53 / 201)$ patients had received previous antifungal treatment, and paediatric patients accounted for $71.4 \%(25 / 35)$ of the total. The underlying comorbidities in adult patients were significantly worse than those in paediatric patients, but the number of underlying conditions in paediatric patients were significantly higher than those in adult patients, and the difference was statistically significant $(P<0.05)$. FCA was the most frequently used empirical antifungal treatment (60/114, 52.6\%). The demographic and clinical characteristics of the patients are summarized in Table 1 and Table 2.

The mean annual incidence of candidaemia was 0.26 / 1000 admissions, including 0.42/1000 paediatric admissions (1.61/1000 neonatal admissions (age $<28$ days), $0.06 / 1000$ infant admissions (28 days $<$ age $<1$ year) and $0.04 / 1000$ child admissions ( 1 year $<$ age $<16$ years) $)$ and $0.24 / 1000$ adult admissions (0.09/1000 surgical admissions, $0.30 / 1000$ medical admissions and 1.64/1000 ICU admissions). According to the Candida species, the incidence of the three most commonly isolated Candida species were as follows: C. albicans, 0.10/1000 admissions; C. glabrata, 0.09/1000 admissions; and C. tropicalis, 0.04/1000 admissions.

The most common species among all Candida species isolates was C. albicans (40.3\%), followed by C. glabrata (36.3\%), C. tropicalis (13.9\%), C. parapsilosis (4.0\%), C. krusei $(3.0 \%)$ and others (2.5\%). The distributions of Candida species in paediatric ( $<16$ years) and adult $(\geq 16$ years) patients are shown in Table 1. In patients aged 0 16 years and $49-65$ years, C. glabrata was the predominant species (51.4 and $41.1 \%$, respectively), but in patients aged $17-49$ and $>65$ years, $C$. albicans was the main species (45.7 and 56.9\%, respectively). The distribution of Candida species in paediatric, surgical, internal medicine and ICU wards is shown in Fig. 1.

The results of in vitro susceptibility testing of Candida strain isolates are summarized in Table 3. All isolates were highly susceptible to AMB (99.0\%) and 5-FC (99.0\%). The resistance rates of ITR, VRC and FCA were 24.919 .4 and $18.5 \%$, respectively. C.tropicalis had the highest antifungal agent resistance rate among the Candida species and was resistant to FCA (39.3\%), ITR (39.3\%) and VRC (42.9\%). The activity of antifungal agents against Candida species was not significantly different in terms of satisfactory outcomes between paediatric and adult patients $(P>0.05)$. The detailed data are shown in Table 3.

The all-cause mortality rate in the 201 patients was $20.4 \%(41 / 201)$. The 7 -day and 30-day mortality rates were $8.5 \%(17 / 201)$ and $17.9 \%(36 / 201)$, respectively. The mortality rates of C. albicans, C. glabrata, C. tropicalis and C. parapsilosis infections were $27.2 \%(22 / 81)$, $16.4 \%(12 / 73), 21.4 \%(6 / 28)$ and $12.5 \%(1 / 8)$, respectively. The mortality rates for paediatric wards, medical wards, surgical wards and ICU wards were $11.4 \%(4 / 35)$, $22.5 \%(20 / 89), 16.1 \%(5 / 31)$ and $26.1 \%(12 / 46)$, respectively. The mortality rates for different age groups were $11.4 \%$ (4/35, $0-16$ years) among paediatric patients and $22.3 \% \quad(37 / 166(>16$ years $), \quad 7.7 \% \quad(4 / 52, \quad 17-49$ years $)$, $19.6 \%(11 / 56,50-65$ years $)$ and $37.9 \%(22 / 58,>65$ years)) among adult patients.

The univariate predictors of poor outcomes due to candidaemia are shown in Table 4. For paediatric 
Table 1 Distribution and incidence of Candida species

\begin{tabular}{|c|c|c|c|c|c|c|c|}
\hline & \multicolumn{7}{|c|}{ Candida species } \\
\hline & Total & C. albicans & C. glabrata & C. tropicalis & C. parapsilosis & C. krusei & others \\
\hline & $\begin{array}{l}(n=201) \\
100.0 \%\end{array}$ & $\begin{array}{l}(n=81) \\
40.3 \%\end{array}$ & $\begin{array}{l}(n=73) \\
36.3 \%\end{array}$ & $\begin{array}{l}(n=28) \\
13.9 \%\end{array}$ & $\begin{array}{l}(n=8) \\
4.0 \%\end{array}$ & $\begin{array}{l}(n=6) \\
3.0 \%\end{array}$ & $\begin{array}{l}(n=5) \\
2.5 \%\end{array}$ \\
\hline \multicolumn{8}{|l|}{ Distribution n(\%) } \\
\hline Paediatric patients ( $\leq 16$ years) & $35(17.4)$ & $16(45.7)$ & $18(51.4)$ & $0(0)$ & $0(0)$ & $0(0)$ & $1(2.9)$ \\
\hline $0-28$ days & $32(15.9)$ & $16(50.0)$ & 15(46.9) & $0(0)$ & $0(0)$ & $0(0)$ & $1(3.1)$ \\
\hline 29 days - 1 year & $1(0.5)$ & $0(0)$ & $1(100.0)$ & $0(0)$ & $0(0)$ & $0(0)$ & $0(0)$ \\
\hline $2-16$ years & $2(1.0)$ & $0(0)$ & $2(100.0)$ & $0(0)$ & $0(0)$ & $0(0)$ & $0(0)$ \\
\hline Adult patients (> 16 years) & 166(82.6) & $65(39.2)$ & $55(33.1)$ & 28(16.9) & $8(4.8)$ & $6(3.6)$ & $4(2.4)$ \\
\hline $17-49$ years & $52(25.9)$ & $21(40.4)$ & $19(36.5)$ & $7(13.5)$ & $1(1.9)$ & $3(5.8)$ & $1(1.9)$ \\
\hline $50-65$ years & $56(27.9)$ & $11(19.6)$ & $23(41.1)$ & $14(25.0)$ & $5(8.9)$ & $1(1.8)$ & $2(3.6)$ \\
\hline$>65$ years & $58(28.8)$ & $33(56.9)$ & $13(22.4)$ & $7(12.1)$ & $2(3.4)$ & $2(3.4)$ & $1(1.7)$ \\
\hline \multicolumn{8}{|l|}{ Gender } \\
\hline Male & $114(56.7)$ & $44(38.6)$ & $39(34.2)$ & $19(16.7)$ & $6(5.3)$ & $2(1.7)$ & $4(3.5)$ \\
\hline Female & $87(43.3)$ & $37(42.5)$ & $34(39.1)$ & $9(10.3)$ & $2(2.3)$ & $4(4.6)$ & $1(1.1)$ \\
\hline \multicolumn{8}{|c|}{ Incidence (episodes/1000 admissions) } \\
\hline 2013 & 0.20 & 0.06 & 0.12 & 0.01 & 0.00 & 0.01 & 0.00 \\
\hline 2014 & 0.22 & 0.09 & 0.10 & 0.03 & 0.00 & 0.00 & 0.01 \\
\hline 2015 & 0.27 & 0.10 & 0.12 & 0.02 & 0.00 & 0.03 & 0.00 \\
\hline 2016 & 0.37 & 0.23 & 0.10 & 0.04 & 0.00 & 0.01 & 0.00 \\
\hline 2017 & 0.32 & 0.10 & 0.14 & 0.07 & 0.01 & 0.00 & 0.00 \\
\hline 2018 & 0.16 & 0.06 & 0.07 & 0.02 & 0.00 & 0.00 & 0.00 \\
\hline 2019 & 0.26 & 0.08 & 0.04 & 0.05 & 0.05 & 0.01 & 0.03 \\
\hline Mean annual incidence & 0.26 & 0.10 & 0.09 & 0.04 & 0.01 & 0.01 & 0.01 \\
\hline
\end{tabular}

*Others include C. guilliermondii (3), C. haemulonii (3) and C. inconspicua (1)

patients with candidaemia, the variables associated with 30-day mortality were as follows: length of hospital stay, respiratory dysfunction, chronic/acute renal failure and septic shock. For adult patients with candidaemia, the variables associated with 30-day mortality were as follows: age, length of hospital stay, respiratory dysfunction, pulmonary infection, cardiovascular disease, chronic/ acute renal failure, other invasive catheters, mechanical ventilation, septic shock, C. albicans infection, concomitant bacterial infection and haematologic (nonmalignant) disease. The results of the multivariate analysis are listed in Table 5. Because the total numbers of paediatric patients (35 patients) and deaths (3 patients) were very small, multivariable logistic regression analysis was not performed for paediatric patients. Respiratory dysfunction and septic shock were independent predictors of 30-day mortality in all patients and adult patients. The length of hospital stay was a protective factor for 30-day mortality in all patients and adult patients, and other invasive catheters were only the protective factor for 30day mortality in all patients. The prognostic factors for 30-day mortality in all patients and adult patients were almost the same, and the independent predictors were the same, with no significant differences (Table 5). In addition, we compared the independent protective factors and independent risk factors in this study with those in other studies and found that the protective factors (length of hospital stay and other invasive catheters) in our study were different from those in other studies, while the independent risk factor of septic shock in our study was also identified in some others studies, but the independent risk factor of respiratory dysfunction was not reported in other studies (Table 6).

\section{Discussion}

This was a 7-year retrospective study of candidaemia in a regional tertiary teaching hospital in Southwest China. We not only analysed the epidemiological characteristics, including the basic information of patients, underlying comorbidities, risk factors, the distributions of Candida species, antifungal agent use, antifungal agent susceptibility results and patient outcomes, but also performed epidemiological comparisons between paediatric patients and adult patients. To our knowledge, this is 
Table 2 Patient characteristics and incidence (episode/1000 admission)

\begin{tabular}{|c|c|c|c|c|}
\hline & $\begin{array}{l}\text { All patients } \\
(n=201) \\
100.0 \%\end{array}$ & $\begin{array}{l}\text { Child patients( } 0-16 \text { years }) \\
(n=35) \\
17.4 \%\end{array}$ & $\begin{array}{l}\text { Adult patients }(>16 \text { years }) \\
(n=166) \\
82.6 \%\end{array}$ & $\mathrm{P}^{*}$ \\
\hline Age (median, range) & $\begin{array}{l}65 \text { years } \\
\text { ( } 1 \text { day, } 92 \text { years) }\end{array}$ & $\begin{array}{l}1 \text { day } \\
\text { (1 day,5 years) }\end{array}$ & $\begin{array}{l}61 \text { years } \\
\text { (18 years, } 92 \text { years) }\end{array}$ & $<0.001$ \\
\hline Gender (male:female) & $114: 87$ & $22: 13$ & $92: 74$ & 0.420 \\
\hline Length of hospital stay(days) & $36.9 \pm 39.5$ & $41.5 \pm 20.9$ & $30.6 \pm 39.6$ & 0.117 \\
\hline \multicolumn{5}{|l|}{ Underlying comorbidities $(n, \%)$} \\
\hline Gastrointestinal perforation & $24(11.9)$ & $2(5.7)$ & $22(13.3)$ & 0.211 \\
\hline Respiratory dysfunction ${ }^{a}$ & $58(28.9)$ & $3(8.6)$ & $55(33.1)$ & 0.004 \\
\hline Pulmonary infection & $100(49.8)$ & $24(68.6)$ & $76(45.8)$ & 0.014 \\
\hline Cardiovascular disease & $86(42.8)$ & $3(8.6)$ & $83(50.0)$ & $<0.001$ \\
\hline Neurological diseases & 78 (38.8) & $24(68.6)$ & $54(32.5)$ & $<0.001$ \\
\hline Gastrointestinal pathology ${ }^{b}$ & $58(28.9)$ & $2(5.7)$ & $43(25.9)$ & 0.001 \\
\hline Chronic/acute liver disease & $49(24.4)$ & $9(25.7)$ & $40(24.1)$ & 0.839 \\
\hline Chronic/acute renal failure ${ }^{c}$ & $91(45.3)$ & $9(25.7)$ & $82(49.4)$ & 0.011 \\
\hline Solid tumour & $15(7.5)$ & $0(0)$ & $15(9.0)$ & 0.065 \\
\hline Haematological malignancy & $11(5.5)$ & $2(5.7)$ & $9(5.4)$ & 0.697 \\
\hline Congenital malformations/syndromes & $6(3.0)$ & $3(8.6)$ & $3(1.8)$ & $<0.001$ \\
\hline Diabetes mellitus & $60(29.9)$ & $0(0)$ & $60(36.1)$ & $<0.001$ \\
\hline Hematologic (nonmalignant) & $29(14.4)$ & $10(28.6)$ & $19(11.4)$ & 0.009 \\
\hline HIV/AIDS & $10(5.0)$ & $0(0)$ & $10(6.0)$ & 0.136 \\
\hline Severe trauma & $17(8.5)$ & $2(5.7)$ & $15(9.0)$ & 0.521 \\
\hline \multicolumn{5}{|l|}{ Risk factors $(n, \%)$} \\
\hline Presence of $C V C^{d}$ & $77(38.3)$ & $7(20.0)$ & $70(42.2)$ & 0.014 \\
\hline Other invasive catheters & 60(29.9) & $5(14.3)$ & $55(33.1)$ & 0.027 \\
\hline Mechanical ventilation & $87(43.3)$ & $21(60.0)$ & $66(39.8)$ & 0.028 \\
\hline Receipt of corticosteroids ${ }^{\mathrm{e}}$ & $42(20.9)$ & $9(25.7)$ & 33(19.9) & 0.440 \\
\hline Total parenteral nutrition & $95(47.3)$ & 18(51.4) & $77(46.4)$ & 0.587 \\
\hline Malnutrition & $55(27.4)$ & $9(25.7)$ & $46(27.7)$ & 0.810 \\
\hline Chemotherapy & $20(10.0)$ & $2(5.7)$ & 18(10.8) & 0.357 \\
\hline Hemodialysis & $30(16.9)$ & $0(0)$ & $30(18.1)$ & 0.006 \\
\hline Abdominal surgery ${ }^{f}$ & 31 (15.4) & $0(0)$ & $31(18.7)$ & 0.005 \\
\hline ICU/PICU/NICU & $81(40.3)$ & $35(100.0)$ & $46(27.7)$ & $<0.001$ \\
\hline Neutropenia ${ }^{9}$ & $16(8.0)$ & $0(0)$ & 16(9.6) & 0.056 \\
\hline Concomitant bacterial infections & $110(54.7)$ & $30(85.7)$ & $80(48.2)$ & $<0.001$ \\
\hline Septic shock & $39(19.4)$ & $3(8.6)$ & $36(21.7)$ & 0.075 \\
\hline Broad-spectrum antibiotics & $179(89.1)$ & $35(100.0)$ & $144(86.7)$ & 0.022 \\
\hline Treatment with antifungal agents & $114(56.7)$ & $27(77.1)$ & $87(52.4)$ & 0.007 \\
\hline C. albicans & $81(40.3)$ & 16(45.7) & 65(39.2) & 0.472 \\
\hline C. glabrata & $73(36.3)$ & $18(51.4)$ & $55(33.1)$ & 0.041 \\
\hline C. tropicalis & $28(13.9)$ & $0(0)$ & $28(16.9)$ & 0.009 \\
\hline Death & $41(20.4)$ & $4(11.4)$ & $37(22.3)$ & 0.113 \\
\hline \multicolumn{5}{|l|}{ Incidence(n,episodes/1000 admissions) } \\
\hline 2013 & $14(0.20)$ & $0(0.0)$ & $14(0.24)$ & 0.248 \\
\hline 2014 & 23(0.22) & $1(0.09)$ & $22(0.24)$ & 0.507 \\
\hline
\end{tabular}


Table 2 Patient characteristics and incidence (episode/1000 admission) (Continued)

\begin{tabular}{|c|c|c|c|c|}
\hline & All patients & Child patients(0-16 years) & Adult patients(> 16 years) & $P^{*}$ \\
\hline & $\begin{array}{l}(n=201) \\
100.0 \%\end{array}$ & $\begin{array}{l}(n=35) \\
17.4 \%\end{array}$ & $\begin{array}{l}(n=166) \\
82.6 \%\end{array}$ & \\
\hline 2015 & $29(0.27)$ & $6(0.53)$ & $23(0.24)$ & 0.117 \\
\hline 2016 & $42(0.37)$ & $12(1.07)$ & $30(0.29)$ & 0.001 \\
\hline 2017 & $38(0.32)$ & $12(1.06)$ & $26(0.24)$ & $<0.00$ \\
\hline 2018 & 20(0.16) & $3(0.21)$ & $17(0.15)$ & 0.480 \\
\hline 2019 & $35(0.26)$ & $1(0.08)$ & $34(0.27)$ & 0.252 \\
\hline Mean annual incidence & $201(0.26)$ & $35(0.42)$ & $166(0.24)$ & 0.002 \\
\hline
\end{tabular}

* Statistical results of demographic characteristics of pediatric and adult patients

${ }^{a}$ Includes the following diseases: chronic obstructive pulmonary disease and acute respiratory distress syndrome

${ }^{b}$ Includes the following diseases: cholecystitis, pancreatitis, and peritonitis

${ }^{c}$ Chronic/Acute renal failure is the permanent or sudden and often temporary loss of kidney function with $\mathrm{N}$ waste retention and hypourocrinia

${ }^{\mathrm{d}}$ CVC central venous catheter

$e_{a}$ dose equivalent to the prednisone dosage of $0.3 \mathrm{mg} / \mathrm{kg} /$ day for at least 14 days

including: gastrointestinal perforations, severe acute pancreatitis and complex ventral hernia

${ }^{g}$ Neutropenia is the absolute neutrophil count, that is, $<500$ cells $/ \mu \mathrm{l}$

the first epidemiological comparative study of candidaemia between paediatric and adults patients in Southwest China, which provides reference data for the prevention and treatment of candidaemia in paediatric and adult patients.

Our data showed that there was no significant difference in the sex ratio, length of hospital stay or mortality between adult and paediatric patients $(P>0.05)$. However, the proportions of underlying comorbidities in paediatric patients, including pulmonary infection, neurological diseases, congenital malformations/ syndromes and haematologic (nonmalignant) disease, were higher than those in adult patients $(P<0.05)$, and the other proportions in adult patients were similar or higher than those in paediatric patients (Table 2). Among the risk factors, only $\mathrm{CVC}$, other invasive catheters and abdominal surgery in adult patients had higher risks than those in paediatric patients $(P<0.05)$, and other risk factors in children had higher or similar risks as those in adult patients (Table 2). Fewer univariate predictors of poor outcomes were identified for paediatric patients than for adults patients (4 vs 11 predictors),

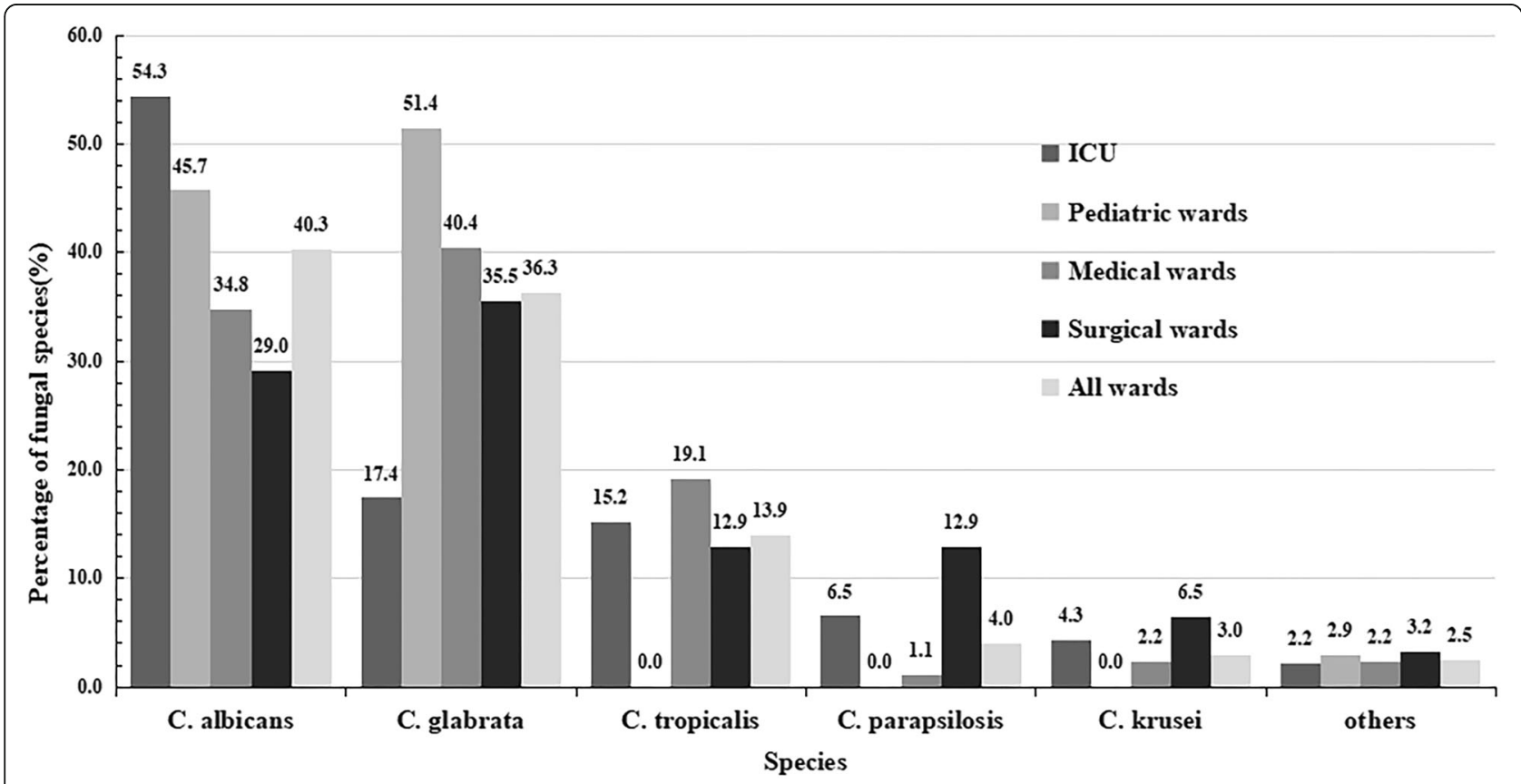

Fig. 1 Distribution of the fungal species according to different wards. FootNote: Others include C. guilliermondii (3), C. haemulonii (1) and C. inconspicua (1) 
Table 3 In vitro antifungal susceptibility testing of 201 clinical isolates into 5 antifungal agents

\begin{tabular}{|c|c|c|c|c|c|}
\hline \multirow{2}{*}{$\begin{array}{l}\text { Species } \\
\text { (No of isolates) }\end{array}$} & \multirow{2}{*}{$\begin{array}{l}\text { Antifungal } \\
\text { agent }\end{array}$} & \multicolumn{4}{|l|}{ Resistant $\mathrm{n}(\%)^{\mathrm{e}}$} \\
\hline & & Children (35) & Adults(166) & total & $P^{c}$ \\
\hline \multirow[t]{5}{*}{ Candida albicans(81) } & Amphotericin B & 0 & 0 & $0^{\mathrm{b}}$ & - \\
\hline & Flucytosine & 0 & $1(1.5)$ & $1(1.2)^{b}$ & 0.618 \\
\hline & Fluconazole & $2(12.5)$ & $17(26.2)$ & $19(23.5)$ & 0.248 \\
\hline & Itraconazole & $8(50.0)$ & $22(33.8)$ & $30(37.0)^{\mathrm{b}}$ & 0.231 \\
\hline & Voriconazole & $4(25.0)$ & 20(30.8) & $24(29.6)^{b}$ & 0.651 \\
\hline \multirow[t]{5}{*}{ Candida glabrata(73) } & Amphotericin B & $1(5.6)$ & 0 & $1(1.4)^{b}$ & 0.078 \\
\hline & Flucytosine & 0 & 0 & $0^{\mathrm{b}}$ & - \\
\hline & Fluconazole & $1(5.6)$ & $5(9.1)$ & $6(8.2)$ & 0.635 \\
\hline & Itraconazole & $2(11.1)$ & $5(9.1)$ & $7(9.6)^{b}$ & 0.801 \\
\hline & Voriconazole & $1(5.6)$ & $2(3.6)$ & $3(4.1)^{b}$ & 0.722 \\
\hline \multirow[t]{5}{*}{ C.tropicalis(28) } & Amphotericin B & 0 & $1(3.6)$ & $1(3.6)^{b}$ & - \\
\hline & Flucytosine & 0 & $1(3.6)$ & $1(3.6)^{b}$ & - \\
\hline & Fluconazole & 0 & $11(39.3)$ & $11(39.3)^{b}$ & - \\
\hline & Itraconazole & 0 & $11(39.3)$ & $11(39.3)^{b}$ & - \\
\hline & Voriconazole & 0 & $12(42.9)$ & $12(42.9)^{b}$ & - \\
\hline \multirow[t]{5}{*}{ C. krusei(6) } & Amphotericin B & 0 & 0 & 0 & - \\
\hline & Flucytosine & 0 & 0 & 0 & - \\
\hline & Fluconazole $^{a}$ & - & - & - & - \\
\hline & Itraconazole & 0 & $1(16.7)$ & $1(16.7)^{b}$ & - \\
\hline & Voriconazole & 0 & $1(16.7)$ & $1(16.7)^{\mathrm{b}}$ & - \\
\hline \multirow[t]{5}{*}{ All of isolates (201) } & Amphotericin B & $1(2.9)$ & $1(0.6)$ & $2(1.0)$ & 0.222 \\
\hline & Flucytosine & $0(0)$ & $2(1.2)$ & $2(1 . .0)$ & 0.513 \\
\hline & Fluconazole ${ }^{a}$ & $3(8.6)$ & $33(20.6)$ & $36(18.5)$ & 0.096 \\
\hline & Itraconazole & 10(28.6) & $40(24.1)$ & $50(24.9)$ & 0.578 \\
\hline & Voriconazole & $5(14.3)$ & $34(20.5)$ & 39(19.4) & 0.400 \\
\hline
\end{tabular}

MIC minimal inhibitory concentration

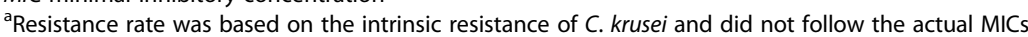

${ }^{\mathrm{b}}$ The breakpoints of Candida spp. according to the manufacturer's instructions of ATB FUNGUS 3 system

'The difference of resistance rate between children and adults was analyzed by chi square test

${ }^{e}$ C. parapsilosis and others isolates (C. guilliermondii (3), C. haemulonii (1) and C. inconspicua (1)) were susceptible to five antifungal agents (100\%), so the results were not listed

as shown in Table 4. This situation has not been clearly shown in other studies, and more epidemiological investigations are needed for confirmation. The incidence of candidaemia among paediatric patients was significantly higher than that among adults $(P<0.05)$ (Table 2$)$; however, no significant difference in mortality was found between paediatric patients and adult patients $(P>0.05)$ (Table 2) in contrast to other studies [16, 17].

Our data showed that the median age of patients with candidaemia and the proportion of males were similar to those in other studies [8,18-23]. Moreover, our study showed that the patients with candidaemia were hospitalised mostly in internal medicine wards, which is different from other studies that reporting hospitalisation mainly in ICU wards $[8,22,24-27]$, but similar to other studies [28-31]. This phenomenon may be related to the demographic characteristics of the inpatients in our hospital, most of whom had more than two underlying diseases and were hospitalised in internal medicine wards. However, the incidence of candidaemia was still the highest in the ICU, similar to other studies [8, 30-34]. In accordance with other studies [17-19, 24, 25, 30, 32, $35,36], C$. albicans was the most common cause of candidaemia in the whole hospital, but the proportion of non-C. albicans infections was higher than that of $C$. albicans infections. Moreover, the proportions of $C$. glabrata in surgical, internal medicine and paediatric wards were the highest, which is different from other studies in China $[18,19,35-37]$ but similar to other studies in other countries $[4,22,27,29,32]$. This may be due to the large number of elderly patients and the increasing use of azole antifungal agents. 
Table 4 Factors associated with 30-days mortality by univariate analysis in inpatients with candidaemia

\begin{tabular}{|c|c|c|c|c|c|c|c|c|c|}
\hline \multirow[t]{2}{*}{ Variable } & \multicolumn{2}{|c|}{$\begin{array}{l}\text { Adult patients } \\
\text { (>16 years) } \\
\text { 30-days outcome }\end{array}$} & \multirow[t]{2}{*}{$P$-value } & \multicolumn{2}{|c|}{$\begin{array}{l}\text { Child patients ( } 0-16 \text { years) } \\
30 \text {-days outcome }\end{array}$} & \multirow[t]{2}{*}{$P$-value } & \multicolumn{2}{|l|}{$\begin{array}{l}\text { All patients } \\
\text { 30-days outcome }\end{array}$} & \multirow[t]{2}{*}{$P$-value } \\
\hline & $\begin{array}{l}\text { Survived } \\
(n=133)\end{array}$ & $\begin{array}{l}\text { Died } \\
(n=33)\end{array}$ & & $\begin{array}{l}\text { Survived } \\
(n=32)\end{array}$ & $\begin{array}{l}\text { Died } \\
(n=3)\end{array}$ & & $\begin{array}{l}\text { Survived }(n= \\
165)\end{array}$ & $\begin{array}{l}\text { Died } \\
(n=36)\end{array}$ & \\
\hline Median age(range) & $\begin{array}{l}60 \text { years } \\
(18,92 \text { years })\end{array}$ & $\begin{array}{l}67 \text { years } \\
(29,86 \text { years })\end{array}$ & 0.001 & $\begin{array}{l}1 \text { days } \\
\text { (1 day, } 5 \text { year) }\end{array}$ & $\begin{array}{l}1 \text { days } \\
\text { ( } 1 \text { day, } 1 \text { day })\end{array}$ & 0.585 & $\begin{array}{l}52 \text { years } \\
\text { ( } 1 \text { day, } 92 \text { years) }\end{array}$ & $\begin{array}{l}65 \text { years } \\
\text { ( } 1 \text { day, } 86 \text { years) }\end{array}$ & 0.001 \\
\hline Gender (male:female) & $72: 61$ & $20: 13$ & 0.503 & 19:13 & $3: 0$ & 0.164 & $91: 74$ & $23: 13$ & 0.557 \\
\hline $\begin{array}{l}\text { Length of hospital } \\
\text { stay(days) }\end{array}$ & $35.3 \pm 42.8$ & $11.7 \pm 9.2$ & 0.002 & $43.8 \pm 20.2$ & $16.7 \pm 8.5$ & 0.029 & $36.9 \pm 39.5$ & $12.1 \pm 9.1$ & $<0.001$ \\
\hline \multicolumn{10}{|l|}{$\begin{array}{l}\text { Underlying comorbidities } \\
(n, \%)\end{array}$} \\
\hline $\begin{array}{l}\text { Gastrointestinal } \\
\text { perforation }\end{array}$ & 15(11.3) & $7(21.2)$ & 0.132 & $2(6.3)$ & $0(0)$ & 0.656 & $17(10.3)$ & $7(19.4)$ & 0.125 \\
\hline $\begin{array}{l}\text { Respiratory } \\
\text { dysfunction }\end{array}$ & $28(21.1)$ & $27(81.8)$ & $\begin{array}{l}< \\
0.001\end{array}$ & $1(3.1)$ & $2(66.7)$ & $\begin{array}{l}< \\
0.001\end{array}$ & $29(17.6)$ & $29(80.6)$ & $<0.001$ \\
\hline Pulmonary infection & $54(40.6)$ & $22(66.7)$ & 0.007 & $22(68.8)$ & $2(66.7)$ & 0.941 & $76(46.1)$ & $24(66.7)$ & 0.025 \\
\hline Cardiovascular disease & $56(42.1)$ & $27(81.8)$ & $\begin{array}{l}< \\
0.001\end{array}$ & $3(9.4)$ & $0(0)$ & 0.579 & $59(35.8)$ & $27(75.0)$ & $<0.001$ \\
\hline Neurological diseases & $41(30.8)$ & 13(39.4) & 0.347 & $22(68.8)$ & $2(66.7)$ & 0.941 & $63(38.2)$ & $15(41.7)$ & 0.697 \\
\hline $\begin{array}{l}\text { Gastrointestinal } \\
\text { pathology }\end{array}$ & $42(31.6)$ & $14(42.4)$ & 0.238 & $2(6.3)$ & $0(0)$ & 0.656 & $44(26.7)$ & $14(38.9)$ & 0.143 \\
\hline $\begin{array}{l}\text { Chronic/acute liver } \\
\text { disease }\end{array}$ & $30(22.6)$ & 10(30.3) & 0.352 & $9(28.1)$ & $0(0)$ & 0.287 & $39(23.6)$ & $10(27.8)$ & 0.600 \\
\hline $\begin{array}{l}\text { Chronic/acute renal } \\
\text { failure }\end{array}$ & $60(45.1)$ & $22(66.7)$ & 0.027 & $6(18.8)$ & $3(100.0)$ & 0.002 & $66(40.0)$ & $22(61.1)$ & 0.001 \\
\hline $\begin{array}{l}\text { Haematological } \\
\text { malignancy }\end{array}$ & $4(3.0)$ & $3(9.1)$ & 0.120 & $2(6.3)$ & 20 & 0.656 & $6(36.4)$ & $5(13.9)$ & 0.217 \\
\hline Solid tumour & $12(9.0)$ & $3(9.1)$ & 0.990 & $0(0)$ & $0(0)$ & - & $12(7.3)$ & $3(8.3)$ & 0.826 \\
\hline $\begin{array}{l}\text { Severe autoimmune } \\
\text { diseases }\end{array}$ & $12(9.0)$ & $3(9.1)$ & 0.990 & $0(0)$ & $0(0)$ & - & $12(7.3)$ & $3(8.3)$ & 0.826 \\
\hline $\begin{array}{l}\text { Congenital } \\
\text { malformations/ } \\
\text { syndromes }\end{array}$ & 0 & 0 & - & $5(15.6)$ & $1(33.3)$ & 0.365 & $5(3.0)$ & $1(2.8)$ & 0.799 \\
\hline $\begin{array}{l}\text { Hematologic } \\
\text { (nonmalignant) }\end{array}$ & $12(9.0)$ & $7(21.2)$ & 0.049 & $9(28.1)$ & $1(33.3)$ & 0.849 & $21(12.7)$ & $8(22.2)$ & 0.142 \\
\hline Diabetes mellitus & 45(33.8) & 15(45.5) & 0.214 & $0(0)$ & $0(0)$ & - & $45(27.3)$ & $15(41.7)$ & 0.087 \\
\hline HIV/AIDS & $9(6.8)$ & $1(3.0)$ & 0.419 & $0(0)$ & $0(0)$ & - & $9(5.5)$ & $1(2.8)$ & 0.503 \\
\hline Severe trauma & $12(9.0)$ & $3(9.1)$ & 0.990 & $2(6.3)$ & $0(0)$ & 0.565 & $14(8.5)$ & $3(8.3)$ & 0.976 \\
\hline \multicolumn{10}{|l|}{ Risk factors $(n, \%)$} \\
\hline $\begin{array}{l}\text { premature neonates } \leq 36 \\
\text { weeks) }^{\mathrm{a}}\end{array}$ & - & - & - & $28(87.5)$ & $2(66.7)$ & 0.515 & 28(93.3) & $2(100.0)$ & 0.706 \\
\hline $\begin{array}{l}\text { Very low birth weight } \\
\text { neonates }(<1500 \mathrm{~g})^{\mathrm{a}}\end{array}$ & - & - & - & $19(59.4)$ & $2(66.7)$ & 0.886 & $19(63.3)$ & $2(100.0)$ & 0.290 \\
\hline Presence of CVC & $57(42.9)$ & 13(39.4) & 0.718 & $6(18.8)$ & $1(33.3)$ & 0.546 & $63(38.2)$ & $14(38.9)$ & 0.937 \\
\hline $\begin{array}{l}\text { Other invasive } \\
\text { catheters }\end{array}$ & $51(38.3)$ & $4(12.1)$ & 0.004 & $5(15.6)$ & $0(0)$ & 0.460 & $56(33.9)$ & $4(11.1)$ & 0.007 \\
\hline Mechanical ventilation & $45(33.8)$ & $21(63.6)$ & 0.002 & $19(59.4)$ & $2(66.7)$ & 0.805 & $64(38.8)$ & $23(63.9)$ & 0.006 \\
\hline Receipt of corticosteroids & $27(20.3)$ & $6(18.2)$ & 0.785 & $9(18.1)$ & $0(0)$ & 0.287 & $36(21.8)$ & $6(16.7)$ & 0.491 \\
\hline Total parenteral nutrition & $57(42.9)$ & $20(60.6)$ & 0.067 & $17(53.1)$ & $1(33.3)$ & 0.512 & $74(44.8)$ & $21(58.3)$ & 0.142 \\
\hline Malnutrition & $36(27.1)$ & 10(30.3) & 0.710 & $8(25.0)$ & $1(33.3)$ & 0.752 & $44(26.7)$ & 11(30.6) & 0.635 \\
\hline Chemotherapy & 16(12.0) & $2(6.1)$ & 0.324 & $2(6.3)$ & $0(0)$ & 0.656 & $18(10.9)$ & $2(5.6)$ & 0.331 \\
\hline Abdominal surgery & 24(18.0) & $7(21.2)$ & 0.676 & $0(0)$ & $0(0)$ & - & $24(14.5)$ & $7(19.4)$ & 0.461 \\
\hline
\end{tabular}


Table 4 Factors associated with 30-days mortality by univariate analysis in inpatients with candidaemia (Continued)

\begin{tabular}{|c|c|c|c|c|c|c|c|c|c|}
\hline \multirow[t]{2}{*}{ Variable } & \multicolumn{2}{|c|}{$\begin{array}{l}\text { Adult patients } \\
\text { (>16 years) } \\
\text { 30-days outcome }\end{array}$} & \multirow[t]{2}{*}{$P$-value } & \multicolumn{2}{|c|}{$\begin{array}{l}\text { Child patients ( } 0-16 \text { years) } \\
30 \text {-days outcome }\end{array}$} & \multirow[t]{2}{*}{$P$-value } & \multicolumn{2}{|c|}{$\begin{array}{l}\text { All patients } \\
\text { 30-days outcome }\end{array}$} & \multirow[t]{2}{*}{$P$-value } \\
\hline & $\begin{array}{l}\text { Survived } \\
(n=133)\end{array}$ & $\begin{array}{l}\text { Died } \\
(n=33)\end{array}$ & & $\begin{array}{l}\text { Survived } \\
(n=32)\end{array}$ & $\begin{array}{l}\text { Died } \\
(n=3)\end{array}$ & & $\begin{array}{l}\text { Survived ( } n= \\
165)\end{array}$ & $\begin{array}{l}\text { Died } \\
(n=36)\end{array}$ & \\
\hline Hemodialysis & $22(16.5)$ & $8(24.2)$ & 0.303 & $0(0)$ & $0(0)$ & - & $22(13.3)$ & $8(22.2)$ & 0.175 \\
\hline ICU/PICU/NICU & $33(24.8)$ & $13(39.4)$ & 0.094 & $32(100.0)$ & $3(100.0)$ & - & 65(39.4) & $16(44.4)$ & 0.576 \\
\hline Neutropenia $^{9}$ & $13(9.8)$ & $3(9.1)$ & 0.905 & $0(0)$ & $0(0)$ & - & $13(7.9)$ & $3(8.3)$ & 0.927 \\
\hline $\begin{array}{l}\text { Concomitant bacterial } \\
\text { infections }\end{array}$ & $59(44.4)$ & $21(63.6)$ & 0.047 & $27(84.4)$ & $3(100.0)$ & 0.460 & $86(52.1)$ & $24(66.7)$ & 0.112 \\
\hline Septic shock & $8(6.0)$ & $28(84.8)$ & $\begin{array}{l}< \\
0.001\end{array}$ & $1(3.1)$ & $2(66.7)$ & $\begin{array}{l}< \\
0.001\end{array}$ & $9(5.5)$ & $30(83.3)$ & $<0.001$ \\
\hline $\begin{array}{l}\text { Broad-spectrum } \\
\text { antibiotics }\end{array}$ & 115(86.5) & $29(87.9)$ & 0.830 & $32(100.0)$ & $3(100.0)$ & - & 147(89.1) & $32(88.9)$ & 0.972 \\
\hline $\begin{array}{l}\text { Treatment with } \\
\text { antifungal agents }\end{array}$ & $71(53.4)$ & $16(48.5)$ & 0.614 & $24(75.0)$ & $3(100.0)$ & 0.324 & $95(57.6)$ & 19(52.8) & 0.599 \\
\hline \multicolumn{10}{|l|}{ Species, n (\%) } \\
\hline C. albicans & $47(35.3)$ & $18(54.5)$ & 0.043 & $16(50.0)$ & $0(0)$ & 0.096 & $63(38.2)$ & $18(50.0)$ & 0.190 \\
\hline C. glabrata & $46(34.6)$ & $9(27.3)$ & 0.424 & $15(46.9)$ & $3(100.0)$ & 0.078 & $61(37.0)$ & $12(33.3)$ & 0.681 \\
\hline
\end{tabular}

ICU intensive care unit; PICU pediatric intensive care unit, NICU neonatal intensive care unit, CVC central venous catheter

${ }^{\mathrm{a}}$ Only neonatal cases were analyzed

Our data showed that the incidence of candidaemia increased from 0.20 episodes/1000 admissions in 2013 to 0.37 episodes in 2016 and then dropped to 0.26 between 2017 and 2019. The change in the annual incidence rate was mainly due to the change in the incidence rate in paediatric patients. The reasons may be due to the gradual easing of restrictions of China's two-child policy since 2013. The number of geriatric pregnant women has increased annually, resulting in an increase in the incidence of neonatal diseases. The change trend was similar to that reported by Oeser et al. [38]. The overall morbidity and 30-day mortality in ICUs and hospitals in

Table 5 Factors associated with 30-days mortality by multivariate analysis ${ }^{a}$

\begin{tabular}{|c|c|c|c|c|c|c|}
\hline \multirow[t]{2}{*}{ Variable } & \multicolumn{3}{|l|}{ All patients } & \multicolumn{3}{|c|}{ Adult patients } \\
\hline & Odds ratio & 95\% confidence interval & $P$-value & Odds ratio & 95\% confidence interval & $P$-value \\
\hline Median age & 1.02 & $0.973-1.065$ & 0.444 & 1.03 & $0.957-1.109$ & 0.427 \\
\hline Length of hospital stay(days) & 0.88 & $0.809-0.964$ & 0.005 & 0.89 & $0.802-0.99$ & 0.032 \\
\hline Respiratory dysfunction & 13.78 & $2.254-84.198$ & 0.005 & 22.57 & $2.014-252.84$ & 0.011 \\
\hline Pulmonary infection & 0.68 & $0.125-3.693$ & 0.655 & 0.98 & $0.142-6.743$ & 0.982 \\
\hline Cardiovascular disease & 0.65 & $0.088-4.787$ & 0.672 & 3.36 & $0.269-41.933$ & 0.347 \\
\hline Chronic/acute renal failure & 2.50 & $0.464-13.425$ & 0.287 & 1.19 & $0.191-7.392$ & 0.854 \\
\hline Other invasive catheters & 0.04 & $0.002-0.695$ & 0.028 & 0.04 & $0.001-1.233$ & 0.066 \\
\hline Mechanical ventilation & 4.59 & $0.554-37.999$ & 0.158 & 12.56 & $0.981-160.793$ & 0.052 \\
\hline Septic shock & 99.97 & 11.997-832.995 & $<0.001$ & 89.72 & $10.161-792.184$ & $<0.001$ \\
\hline Diabetes mellitus ${ }^{\mathrm{b}}$ & 0.12 & $0.013-1.038$ & 0.054 & - & - & - \\
\hline C. Albicans ${ }^{b}$ & - & - & - & 3.16 & $0.391-25.505$ & 0.281 \\
\hline Concomitant bacterial infection ${ }^{b}$ & - & - & - & 4.97 & $0.42-58.742$ & 0.204 \\
\hline Hematologic (nonmalignant) ${ }^{\mathrm{b}}$ & - & - & - & 0.27 & $0.018-4.057$ & 0.346 \\
\hline Total parenteral nutrition ${ }^{b}$ & - & - & - & 0.06 & $0.004-1.06$ & 0.055 \\
\hline ICU/PICU/NICU ${ }^{\mathrm{b}}$ & - & - & - & 0.31 & $0.029-3.259$ & 0.328 \\
\hline
\end{tabular}

ICU intensive care unit; PICU pediatric intensive care unit, NICU neonatal intensive care unit

a Because the total number of pediatric patients(35 patients) and deaths ( 3 patients) were very small, multivariable logistic regression analysis was not performed in pediatric patients

${ }^{\mathrm{b}}$ Biologically plausible variables with a value of $P>0.1$ according to the univariate analyses were not included in the multiple logistic regression model 
Table 6 Protective factor and predictors of 30-day mortality in others studies

\begin{tabular}{|c|c|c|c|c|c|c|c|c|}
\hline Authors & $\begin{array}{l}\text { Country } \\
\text { or region }\end{array}$ & $\begin{array}{l}\text { study } \\
\text { period }\end{array}$ & study design & samples & $\begin{array}{l}\text { No of } \\
\text { samples }\end{array}$ & Protective factor & $\begin{array}{l}\text { Predictors of 30-day } \\
\text { mortality }\end{array}$ & Reference \\
\hline Ma et al & China & 2009-2011 & $\begin{array}{l}\text { Retrospective, } \\
\text { observational, } \\
\text { single-center study }\end{array}$ & $\begin{array}{l}\text { Candidemia(( } 130 \\
\text { adults } \\
\text { and } 3 \text { children < } \\
15 \text { years patients })\end{array}$ & 133 & & Presence of CVC & 37 \\
\hline Cortes et al & Colombia & $2008-2009$ & $\begin{array}{l}\text { Retrospective, } \\
\text { observational, } \\
\text { multicenter study } \\
\text { (seven tertiary-care } \\
\text { hospitals) }\end{array}$ & $\begin{array}{l}\text { Candidemia }(9 \\
\text { days to } 87 \text { years } \\
\text { patients) }\end{array}$ & 131 & Fluconazole therapy & $\begin{array}{l}\text { Age, the presence of } \\
\text { shock at the time of } \\
\text { Candida detection }\end{array}$ & 40 \\
\hline Wang et al & China & $2008-2010$ & $\begin{array}{l}\text { Retrospective, } \\
\text { multicentre } \\
\text { study } \\
\text { (4 tertiary general } \\
\text { hospitals) }\end{array}$ & $\begin{array}{l}\text { Candidemia(> } 16 \\
\text { years patients) }\end{array}$ & 147 & $\begin{array}{l}\text { Antifungal therapy } \\
\text { administered before } \\
\text { microbiological } \\
\text { documentation }\end{array}$ & $\begin{array}{l}\text { Absence of antifungal } \\
\text { therapies, receipt of } \\
\text { mechanical ventilation } \\
\text { and APACHE II score } \geq \\
20\end{array}$ & 41 \\
\hline Tedeschi et al & Italy & 2012-2013 & $\begin{array}{l}\text { Retrospective, } \\
\text { observational, } \\
\text { multicenter cohort } \\
\text { study( } 39 \text { hospitals) }\end{array}$ & $\begin{array}{l}\text { Candidemia } \\
\text { (adult patients) }\end{array}$ & 232 & $\begin{array}{l}\text { Central-venous-catheter } \\
\text { removal and adequate } \\
\text { and timely(within } 72 \mathrm{~h} \text { of } \\
\text { drawing blood cultures) } \\
\text { therapy }\end{array}$ & $\begin{array}{l}\text { Chronic-obstructive- } \\
\text { pulmonary-disease and } \\
\text { isolation of C. tropicalis }\end{array}$ & 42 \\
\hline Li et al & China & 2010-2014 & $\begin{array}{l}\text { Retrospective, } \\
\text { observational, } \\
\text { single-center study }\end{array}$ & $\begin{array}{l}\text { Candidemia(> } 18 \\
\text { years patients) }\end{array}$ & 190 & $\begin{array}{l}\text { Proven catheter-related } \\
\text { candidemia }\end{array}$ & $\begin{array}{l}\text { Severe sepsis or septic } \\
\text { shock }\end{array}$ & 35 \\
\hline Gonzalez-Lara et al & Mexico & 2008-2014 & $\begin{array}{l}\text { Retrospective, } \\
\text { laboratory-based sur- } \\
\text { vey study(two tertiary- } \\
\text { care centers hospitals) }\end{array}$ & $\begin{array}{l}\text { Candidemia(all } \\
\text { patients) }\end{array}$ & 149 & $\begin{array}{l}\text { Early CVC withdrawal } \\
\text { andempirical antifungal } \\
\text { therapy }\end{array}$ & $\begin{array}{l}\text { Severe sepsis and } \\
\text { previous diagnosis of } \\
\text { cirrhosis }\end{array}$ & 43 \\
\hline Jia et al & China & $2011-2016$ & $\begin{array}{l}\text { Retrospective, } \\
\text { observational, } \\
\text { multicenter study ( } 3 \\
\text { hospitals) }\end{array}$ & $\begin{array}{l}\text { Candidemia(15- } \\
90 \text { years patients) }\end{array}$ & 198 & & $\begin{array}{l}\text { ICU admission, } \\
\text { catheter- } \\
\text { relatedcandidemia, as- } \\
\text { cites, septic shock and } \\
\text { concomitant bacterial } \\
\text { infection }\end{array}$ & 18 \\
\hline Ortega-Loubon et al & Spain & $2007-2016$ & $\begin{array}{l}\text { Retrospective, } \\
\text { observational, single- } \\
\text { cente study }\end{array}$ & $\begin{array}{l}\text { Candidemia(> } 18 \\
\text { years patients) }\end{array}$ & 296 & & $\begin{array}{l}\text { Prolonged mechanical } \\
\text { ventilation, age and } \\
\text { low lymphocyte count }\end{array}$ & 23 \\
\hline Kato et al & Japan & 2011-2016 & $\begin{array}{l}\text { Retrospective, } \\
\text { observational, } \\
\text { multicenter study ( } 5 \\
\text { hospitals) }\end{array}$ & $\begin{array}{l}\text { Candidemia(all } \\
\text { patients) }\end{array}$ & 289 & $\begin{array}{l}\text { Follow-up blood culture, } \\
\text { empiric treatment with } \\
\text { fluconazole }\end{array}$ & $\begin{array}{l}\text { Age }>65 \text { years and } \\
\text { SOFA score } \geq 6\end{array}$ & 5 \\
\hline Ala-Houhala et al & Finland & $2007-2016$ & $\begin{array}{l}\text { Retrospective, } \\
\text { observational } \\
\text { study ( } 2 \text { hospitals) }\end{array}$ & $\begin{array}{l}\text { Candidemia(> } 18 \\
\text { years patients) }\end{array}$ & 374 & & $\begin{array}{l}\text { Severity of underlying } \\
\text { illnesses, ICU stay at the } \\
\text { onset of candidemia } \\
\text { and age }>65 \text { years }\end{array}$ & 4 \\
\hline Medeiros et al & Brazil & $2011-2016$ & $\begin{array}{l}\text { Retrospective, single- } \\
\text { center, observational } \\
\text { cohort study }\end{array}$ & $\begin{array}{l}\text { Nosocomial } \\
\text { candidemia }\end{array}$ & 68 & & $\begin{array}{l}\text { Older age, severe } \\
\text { sepsis and hypotension }\end{array}$ & 25 \\
\hline Santolaya et al & Chile & 2013-2017 & $\begin{array}{l}\text { Prospective, } \\
\text { observational } \\
\text { multicenter, } \\
\text { laboratory-based sur- } \\
\text { vey study( } 26 \text { tertiary } \\
\text { care hospitals) }\end{array}$ & $\begin{array}{l}\text { Candidemia(all } \\
\text { patients) }\end{array}$ & 780 & & $\begin{array}{l}\text { Mechanical ventilation } \\
\text { and previous use of } \\
\text { corticosteroids }\end{array}$ & 17 \\
\hline Alkharashi et al & $\begin{array}{l}\text { Saudi } \\
\text { Arabia }\end{array}$ & $2013-2018$ & $\begin{array}{l}\text { Retrospective, } \\
\text { observational, single- } \\
\text { cente study }\end{array}$ & $\begin{array}{l}\text { Candidemia(> } 18 \\
\text { years patients) }\end{array}$ & 324 & & $\begin{array}{l}\text { Use of broad-spectrum } \\
\text { antibiotics and use of } \\
\text { central venous } \\
\text { catheters }\end{array}$ & 24 \\
\hline Xiao et al & China & $2011-2017$ & $\begin{array}{l}\text { Retrospective, } \\
\text { observational, single- } \\
\text { cente study }\end{array}$ & $\begin{array}{l}\text { Candidemia(26- } \\
91 \text { years patients) }\end{array}$ & 82 & & $\begin{array}{l}\text { GCS score, P/F ratio, } \\
\text { MAP }\end{array}$ & 36 \\
\hline This study & China & 2013-2019 & $\begin{array}{l}\text { Retrospective, } \\
\text { observational, single- } \\
\text { cente, cohort study }\end{array}$ & $\begin{array}{l}\text { Candidemia }(0-5 \\
\text { years and }>16 \\
\text { years patients })\end{array}$ & 201 & $\begin{array}{l}\text { Length of hospital } \\
\text { stay(days) }\end{array}$ & $\begin{array}{l}\text { Respiratory dysfunction } \\
\text { and Septic shock }\end{array}$ & $\begin{array}{l}\text { This } \\
\text { study }\end{array}$ \\
\hline
\end{tabular}

CVC central venous catheter; APACHE Acute Physiology and Chronic Health Evaluation; ICU Intensive care unit; SOFA Sequential Organ Failure Assessment; GCS Glasgow Coma Scale; P/F ratio PaO2/FiO2 ratio; MAP Mean arterial pressure 
this study were similar to those in another hospital in this region of China [18], but lower than those in hospitals in other regions of China $[35,37]$ and other countries $[5,8,16,20,21,23,25,30]$. The overall mortality rate of candidaemia has been reported to be $20-49 \%$ globally [39], and the mortality rate was $20.4 \%$ in our hospital, which is low compared to the global rate. This may be because the demographic characteristics and underlying diseases of patients in this region are different from those in other regions or countries, and few severe patients were admitted to our hospital.

With regard to resistance, resistance to FCA, ITR and VRC were common in C. albicans and non-C. albicans species (Table 3). In our study, AMB and 5-FC were highly active against all Candida species. In paediatric patients, the resistance rate of ITR was higher than that in adult patients, but the resistance rates of FCA and VRC were lower than those in adult patients; however, and the resistance rate of Candida species was no significant difference in satisfaction between paediatric and adult patients $(P>0.05)$. Moreover, FCA was highly active against all Candida species in paediatric patients and could be used in paediatric patients with candidaemia as a first-line agent. In the whole hospital, the resistance rate to azole was higher than those reported in other regions $[18,19,36]$ and countries [17, 19, 25, 29, 30, 34]. This may be related to the long-term use of empirical prophylactic drugs by clinicians. Therefore, it was necessary to conduct an epidemiological analysis of antifungal agent susceptibility and guide clinicians to choose the rational antifungal agents to avoid the continuous increase in resistance rates.

In this study, septic shock was an independent predictor of 30-day mortality; which has been reported in many other studies $[18,35]$. However, the other factors reported here have rarely been reported in other studies $[35,40-43]$, possibly because the demographic characteristics, underlying diseases and risk factors of the patients in our study were different from those in other studies; which may be the reason that the independent predictors and protective factors in this study were different from those in other studies [5, 35, 40-43]. The independent predictors and protective factors in different regions and countries are shown in Table 6.

The limitations of this study must be acknowledged. First, this was a single-centre retrospective study, and the total number of patients(166 adult and 35 paediatric patients) were small. Our data might be influenced by the number of patients, the level of medical intervention, and the distribution of patient types. Second, due to technical limitations of the clinical microbiology laboratory and the impact of hospital policies, no data on echinocandins were available in our hospital. Therefore, the results may not be generalizable to all patients with candidaemia in China.

\section{Conclusion}

C. albicans was the main Candida species, but C. glabrata has become the second most common species in this region. FCA was the main antifungal agent for paediatric patients. AMB and 5-FC were highly active against all Candida species. The morbidity and mortality rates in elderly patients were the highest. Respiratory dysfunction and septic shock were independent predictors of 30-day mortality. Further multi-centre studies on candidaemia in different geographical regions in all patients should be conducted to help infection specialists assess the distribution and trends in patients with suspected fungal infections.

\section{Abbreviations}

BSI: Bloodstream infection; ICU: Intensive care unit; PICU: Paediatric intensive care unit; NICU: Neonatal intensive care unit; USA: United States of America; ATCC: American type culture collection; MALDI-TOF MS: Matrix-assisted laser desorption/ionization-time of flight mass spectroscopy; FCA: Fluconazole; ITR: Itraconazole; AMB: Amphotericin B; VRC: Voriconazole; 5-FC: Flucytosine; CVC: Central venous catheter; MIC: Minimal inhibitory concentration; OR: Odds ratio; Cl: Confidence interval

\section{Acknowledgements}

We thank Yanhan Li in the medical records room for guiding us in reviewing the electronic medical records. We have asked for American Journal Experts (AJE, www.aje.com) for its linguistic assistance during the preparation of this revised manuscript.

\section{Authors' contributions}

ZRZ, YHD and JBL designed the study and drafted the manuscript. ZRZ, YHD, KY, JBL, GRL and JD collected the data. ZRZ,YHD, GT GRL and JD andanalyzed the data; ZRZ,YHD and GT wrote the paper. All authors have read approved the final manuscript.

\section{Funding}

This work was supported by the Science and Technology Project of Science and Technology Department of Sichuan Province (No. 2018DJPT0011), the Science and Technology Project of Health and Family Planning Commission of Sichuan Province (No. 17PJ506) and the Affiliated Hospital of Luzhou Medical College Science Foundation (No. 14035). The funder had no role in study design, data collection, and analysis, decision to publish, or preparation of the manuscript.

\section{Availability of data and materials}

The data set supporting the conclusions in this article is available from the corresponding author on reasonable request.

\section{Ethics approval and consent to participate}

The study protocol was approved by the Ethics Committee of the Affiliated Hospital of Southwest Medical University (project no. KY2020043). This is a retrospective study. The need for informed consent was waived by the Clinical Research Ethics Committee.

\section{Consent for publication}

Not applicable.

\section{Competing interests}

The authors declare that they have no competing interests.

Received: 20 April 2020 Accepted: 5 August 2020

Published online: 14 August 2020

\section{References}

1. Kullberg BJ, Arendrup MC. Invasive Candidiasis. N Engl J Med. 2015;373(15): 1445-56. 
2. Antinori S, Milazzo L, Sollima S, et al. Candidemia and invasive candidiasis in adults: a narrative review. Eur J Intern Med. 2016;34:21-8.

3. Tsay SV, Mu Y, Williams S, et al. Burden of Candidemia in the United States, 2017. Clin Infect Dis. 2020. https://doi.org/10.1093/cid/ciaa193.

4. Ala-Houhala M, Valkonen M, Kolho E, et al. Clinical and microbiological factors associated with mortality in candidemia in adult patients 2007-2016. Infect Dis (Lond). 2019;51(11-12):824-30.

5. Kato $H$, Yoshimura $Y$, Suido $Y$, et al. Mortality and risk factor analysis for Candida blood stream infection: a multicenter study. J Infect Chemother. 2019;25(5):341-5.

6. Ghazi S, Rafei R, Osman M, et al. The epidemiology of Candida species in the Middle East and North Africa. J Mycol Med. 2019;29(3):245-52.

7. Falagas ME, Roussos N, Vardakas KZ. Relative frequency of albicans and the various non-albicans Candida spp among candidemia isolates from inpatients in various parts of the world: a systematic review. Int J Infect Dis. 2010;14(11):e954-66

8. Lausch KR, Sogaard M, Rosenvinge FS, et al. High incidence of candidaemia in a nationwide cohort: underlying diseases, risk factors and mortality. Int J Infect Dis. 2018;76:58-63.

9. McCarty TP, Pappas PG. Invasive Candidiasis. Infect Dis Clin North Am. 2016; 30(1):103-24.

10. Strollo S, Lionakis MS, Adjemian J, et al. Epidemiology of hospitalizations associated with invasive candidiasis, United States, 2002-2012. Emerg Infect Dis. 2016;23(1):7-13.

11. Chen $M, X u Y$, Hong $N$, et al. Epidemiology of fungal infections in China. Front Med. 2018;12(1):58-75.

12. Zhang Y, Wang F, Expert Group of the Summit Forum on diagnosis and treatment Strategy of Candidiasis CMA. The diagnosis and treatment of Candidiasis:the expert consensus. Chin J Infect Chemother(Chin). 2011;11(2): 81-95 (http://www.cjic.com.cn/mulu112.htm).

13. Cuenca-Estrella M, Verweij PE, Arendrup MC, et al. ESCMID* guideline for the diagnosis and management of Candida diseases 2012: diagnostic procedures. Clin Microbiol Infect. 2012;18(Suppl 7):9-18.

14. Ullmann AJ, Cornely OA, Donnelly JP, et al. ESCMID* guideline for the diagnosis and management of Candida diseases 2012: developing European guidelines in clinical microbiology and infectious diseases. Clin Microbiol Infect. 2012;18(Suppl 7):1-8.

15. Pappas PG, Kauffman CA, Andes DR, et al. Clinical practice guideline for the Management of Candidiasis: 2016 update by the Infectious Diseases Society of America. Clin Infect Dis. 2016;62(4):e1-50.

16. Braga PR, Cruz IL, Ortiz I, et al. Secular trends of candidemia at a Brazilian tertiary care teaching hospital. Braz J Infect Dis. 2018;22(4):273-7.

17. Santolaya ME, Thompson L, Benadof D, et al. A prospective, multi-center study of Candida bloodstream infections in Chile. PLoS One. 2019;14(3): e0212924.

18. Jia X, Li C, Cao J, et al. Clinical characteristics and predictors of mortality in patients with candidemia: a six-year retrospective study. Eur J Clin Microbiol Infect Dis. 2018;37(9):1717-24.

19. Lin S, Chen R, Zhu S, et al. Candidemia in adults at a tertiary Hospital in China: clinical characteristics, species distribution, resistance, and outcomes. Mycopathologia. 2018;183(4):679-89.

20. Hesstvedt L, Gaustad P, Muller F, et al. The impact of age on risk assessment, therapeutic practice and outcome in candidemia. Infect Dis (Lond). 2019;51(6):425-34.

21. Keighley C, Chen SC, Marriott D, et al. Candidaemia and a risk predictive model for overall mortality: a prospective multicentre study. BMC Infect Dis. 2019;19(1):445.

22. Lindberg E, Hammarstrom H, Ataollahy N, et al. Species distribution and antifungal drug susceptibilities of yeasts isolated from the blood samples of patients with candidemia. Sci Rep. 2019;9(1):3838.

23. Ortega-Loubon C, Cano-Hernandez B, Poves-Alvarez R, et al. The overlooked immune state in Candidemia: a risk factor for mortality. J Clin Med. 2019; 8(10):1512.

24. Alkharashi N, Aljohani S, Layqah L, et al. Candida bloodstream infection: changing pattern of occurrence and antifungal susceptibility over 10 years in a tertiary care Saudi hospital. Can J Infect Dis Med Microbiol. 2019;2019: 2015692.

25. Medeiros MAP, Melo APV, Bento AO, et al. Epidemiology and prognostic factors of nosocomial candidemia in Northeast Brazil: a six-year retrospective study. PLoS One. 2019;14(8):e0221033.
26. Orsetti E, Brescini L, Mazzanti S, et al. Characterisation of candidemia in patients with recent surgery: a 7-year experience. Mycoses. 2019;62(11): 1056-63.

27. Pinto-Magalhaes S, Martins A, Lacerda S, et al. Candidemia in a Portuguese tertiary care hospital: analysis of a 2-year period. J Mycol Med. 2019;29(4): 320-4.

28. Luzzati R, Merelli M, Ansaldi F, et al. Nosocomial candidemia in patients admitted to medicine wards compared to other wards: a multicentre study. Infection. 2016;44(6):747-55.

29. Canela HMS, Cardoso B, Vitali LH, et al. Prevalence, virulence factors and antifungal susceptibility of Candida spp. isolated from bloodstream infections in a tertiary care hospital in Brazil. Mycoses. 2018;61(1):11-21.

30. Papadimitriou-Olivgeris M, Spiliopoulou A, Kolonitsiou F, et al. Increasing incidence of candidaemia and shifting epidemiology in favor of Candida non-albicans in a 9-year period (2009-2017) in a university Greek hospital. Infection. 2019;47(2):209-16.

31. Siopi M, Tarpatzi A, Kalogeropoulou E, et al. Epidemiological trends of Fungemia in Greece with a focus on Candidemia during the recent financial crisis: a 10-year survey in a tertiary care academic hospital and review of literature. Antimicrob Agents Chemother. 2020;64(3):e01516-9. https://doi.org/10.1128/AAC.01516-19.

32. Israel S, Amit S, Israel A, et al. The epidemiology and susceptibility of Candidemia in Jerusalem. Israel Front Cell Infect Microbiol. 2019;9:352.

33. Koehler $\mathrm{P}$, Stecher M, Cornely OA, et al. Morbidity and mortality of candidaemia in Europe: an epidemiologic meta-analysis. Clin Microbiol Infect. 2019;25(10):1200-12.

34. Ryan P, Motherway C, Powell J, et al. Candidaemia in an Irish intensive care unit setting between 2004 and 2018 reflects increased incidence of Candida glabrata. J Hosp Infect. 2019;102(3):347-50.

35. Li Y, Du M, Chen LA, et al. Nosocomial bloodstream infection due to Candida spp. in China: species distribution, clinical features, and outcomes. Mycopathologia. 2016;181(7-8):485-95.

36. Xiao Z, Wang Q, Zhu F, et al. Epidemiology, species distribution, antifungal susceptibility and mortality risk factors of candidemia among critically ill patients: a retrospective study from 2011 to 2017 in a teaching hospital in China. Antimicrob Resist Infect Control. 2019;8:89.

37. Ma CF, Li FQ, Shi LN, et al. Surveillance study of species distribution, antifungal susceptibility and mortality of nosocomial candidemia in a tertiary care hospital in China. BMC Infect Dis. 2013;13:337.

38. Oeser C, Lamagni T, Heath PT, et al. The epidemiology of neonatal and pediatric candidemia in England and Wales, 2000-2009. Pediatr Infect Dis J. 2013;32(1):23-6.

39. Mellinghoff SC, Cornely OA, Jung N. Essentials in Candida bloodstream infection. Infection. 2018;46(6):897-9.

40. Cortes JA, Reyes P, Gomez CH, et al. Clinical and epidemiological characteristics and risk factors for mortality in patients with candidemia in hospitals from Bogota, Colombia. Braz J Infect Dis. 2014;18(6):631-7.

41. Wang $H$, Liu N, Yin M, et al. The epidemiology, antifungal use and risk factors of death in elderly patients with candidemia: a multicentre retrospective study. BMC Infect Dis. 2014;14:609.

42. Tedeschi S, Tumietto F, Giannella M, et al. Epidemiology and outcome of candidemia in internal medicine wards: a regional study in Italy. Eur J Intern Med. 2016;34:39-44

43. Gonzalez-Lara MF, Torres-Gonzalez P, Cornejo-Juarez P, et al. Impact of inappropriate antifungal therapy according to current susceptibility breakpoints on Candida bloodstream infection mortality, a retrospective analysis. BMC Infect Dis. 2017;17(1):753.

\section{Publisher's Note}

Springer Nature remains neutral with regard to jurisdictional claims in published maps and institutional affiliations. 